\title{
Cognitive UWB-OFDM: Pushing Ultrawideband Beyond Its Limit via Opportunistic Spectrum Usage
}

\author{
Hüseyin Arslan and Mustafa E. Şahin
}

\begin{abstract}
:
In a continuously expanding wireless world, the number of radio systems increases every day, and efficient spectrum usage becomes a more significant requirement. Ultrawideband (UWB) and cognitive radio are two exciting technologies that offer new approaches to the spectrum usage. The main objective of this paper is to shed the first light on the marriage of these two important approaches. The strength of orthogonal frequency division multiplexing (OFDM) based UWB in co-existing with licensed systems is investigated. The opportunity concept is defined, and the requirements of the opportunistic spectrum usage are explained. It is proposed to take the UWB-OFDM from the current underlay implementation, and evolve it to a combined underlay and opportunistic spectrum usage technology, leading to cognitive UWB-OFDM. This way, we aim at making UWB more competitive in the wireless market with extended range, higher capacity, better performance, and a wide variety of applications.
\end{abstract}

Index Terms: Cognitive radio, narrowband interference, NBI, opportunistic spectrum usage, spectrum efficiency, ultrawideband, UWB-OFDM.

\section{INTRODUCTION}

Wireless communication systems have been evolving substantially over the last two decades. The explosive growth of the wireless communication market is expected to continue in the future, as the demand for all types of wireless services is increasing. New generations of mobile radio systems aim at providing higher data rates and a wide variety of applications to the mobile users, while serving as many users as possible. However, this goal must be achieved under the constraint of limited available frequency spectrum. Given the high price of the spectrum and its scarcity, radio systems must provide higher capacity and performance through a more efficient use of the available resources. Hence, in order not to limit the economic and technological improvement of the wireless world, it is necessary to find immediate solutions regarding the spectrum usage. Two recent and promising solutions for this problem are ultrawideband (UWB) and cognitive radio [1].

UWB is a promising technology for future short and medium range wireless communication networks with various throughputs including very high data rate applications. It has many tempting features such as low power consumption, significantly low complexity transceivers, and immunity to multipath effects. Cognitive radio, on the other hand, aims at a very efficient spec-

Manuscript received October 20, 2005; approved for publication by ..., ... Editor, January 12, 2006

H. Arslan is with the Electrical Engineering Department, University of South Florida, USA, email: arslan@eng.usf.edu.

M. E. Şahin is with the Electrical Engineering Department, University of South Florida, USA, email: mesahin@eng.usf.edu. trum utilization employing smart wireless devices with awareness, sensing, learning, and adaptation capabilities [2]. As a solution for the spectrum scarcity problem, cognitive radio proposes an opportunistic spectrum usage approach [3], in which frequency bands that are not being used by their primary (licensed) users are utilized by cognitive radios. Both UWB and cognitive radio do not require a license and they do not affect the operation of primary systems. Therefore, they lead to a highly economic and efficient usage of the frequency spectrum.

According to the modern definition, any wireless communication technology that has a bandwidth wider than $500 \mathrm{MHz}$ or a fractional bandwidth ${ }^{1}$ greater than 0.2 can be considered a UWB system. Impulse radio (IR) and orthogonal frequency division multiplexing (OFDM) are the two widely recognized options for implementing UWB.

OFDM, which is the UWB implementation option that we will focus in this paper, has become a very popular technology due to its special features such as robustness against multipath interference, ability to allow frequency diversity with the use of efficient forward error correction (FEC) coding, capability of capturing the multipath energy, and ability to provide high bandwidth efficiency. OFDM can overcome many problems that arise with high bit rate communications, the most significant of which is the time dispersion. In OFDM, the data bearing symbol stream is split into several lower rate streams, and these streams are transmitted on different carriers. Since this increases the symbol period by the number of non-overlapping carriers, multipath echoes affect only a small portion of the neighboring symbols. The remaining inter-symbol interference (ISI) can be removed by cyclically extending the OFDM symbol. In terms of adapting the transmission parameters, OFDM offers many possibilities. Adapting the transmit power, cyclic prefix size, modulation and coding, and the number of sub-carriers are some of these transmission parameters. In addition to adaptation over each packet (as in the case of single carrier systems), OFDM also offers adaptation of parameters for each carrier or over a small group of carriers. In other words, adaptation can be done independently over narrower bands rather than the entire transmission band.

Systems with a spectral allocation similar to UWB are often referred as underlay systems. The severe power limitations on underlay systems restrict their usage to only very short range applications. The main contribution of this paper is that a cognitive UWB-OFDM approach is proposed, which supplements the underlay UWB with an overlay opportunistic spectrum usage approach and aims at increasing the capacity, performance, range, and variety of unlicensed communications. The resis-

\footnotetext{
${ }^{1}$ Fractional bandwidth $=2 \cdot \frac{F_{H}-F_{L}}{F_{H}+F_{L}}$, where $F_{H}$ and $F_{L}$ are the upper and lower edge frequencies, respectively.
} 
tance of UWB-OFDM against licensed narrowband systems is discussed. The opportunity concept is defined, and the opportunity sensing and spectrum shaping features of cognitive UWBOFDM are investigated. The potential behavior of the proposed system under many different scenarios is analyzed, in which either UWB-OFDM or opportunistic spectrum usage may be more preferable.

The organization of the paper is as follows. In Section II, interference related issues are discussed, and narrowband interference (NBI) avoidance in UWB-OFDM is addressed. In Section III, the cognitive radio concept is presented. In Section IV, opportunity is defined, and opportunistic spectrum usage is investigated. In Section V, a cognitive UWB-OFDM system is proposed, and the details regarding its implementation are explained. Finally, in Section VI, conclusions and possible future research topics are given.

\section{INTERFERENCE ISSUES AND COEXISTENCE OF UWB WITH NARROWBAND SYSTEMS}

One of the main concerns in communications system design is to determine how to deal with interference, which can be defined as any kind of signal received beside the desired signal and noise. According to its origin, interference can be categorized as follows.

1. Self-interference, which is caused by the own transmitted signal due to the improper system design.

Examples include inter-symbol, inter-carrier, inter-frame, interpulse, and cross-modulation interferences. Self-interference can be handled by properly designing the system and transceivers.

2. Interference from other users, which can be further categorized as

- Multi-user interference, which is the interference from users using the same system or a similar technology. Co-channel and adjacent channel interferences belong to this category. It can be overcome by a proper multi-access design and/or by employing multi-user detection techniques.

- Interference from other types of technologies. This kind of interference mostly requires interference avoidance or cancelation. It is more difficult to handle compared to multi-user interference, and often it can not be suppressed completely. Narrowband interference is a well-known example for this type of interference.

In this paper, since the focus is specifically on ultrawideband systems, the interference problem will also be considered from a

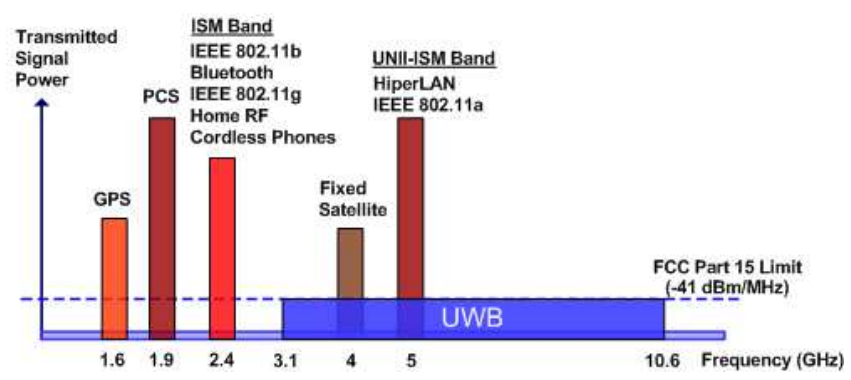

Fig. 1. Spectrum crossover of the narrowband interferers in UWB systems.
UWB point of view. UWB systems operate over extremely wide frequency bands, where various licensed narrowband technologies also exist with much higher power levels (shown in Fig. 1). The transmitted power of UWB devices is controlled by the regulatory agencies (such as the FCC in the United States), so that narrowband systems are affected from UWB signals only at a negligible level. This way, UWB systems are enabled to coexist with these technologies. The influence of narrowband signals on the UWB system, however, can be significant, and in the extreme case, these signals may jam the UWB receiver completely. Even though narrowband systems interfere with only a small fraction of the UWB spectrum, due to their relatively high power with respect to the UWB signal, the performance and capacity of UWB systems can be affected considerably [4]. In the literature, there are several studies showing that the biterror-rate (BER) performance of the UWB receivers is greatly degraded due to the impact of narrowband interference [5]-[9]. The high processing gain of the UWB signal can cope with the narrowband interferers to some extent. However, in many cases, even the large processing gain alone is not sufficient to suppress the effect of the high power interferers. Therefore, either the UWB system needs to avoid transmitting over the frequencies of strong narrowband interferers, or the UWB receivers require to employ NBI suppression techniques to improve the quality of UWB communications.

UWB transmit power level is very limited under the current regulations. Hence, for being able to coexist with licensed systems, resistance to NBI becomes highly significant for the UWB implementation. This fact is a strong motivation behind employing OFDM in UWB applications. OFDM's resistance against NBI depends on its ability to turn the transmission on and off on separate carriers depending on the level of interference temperature. A common NBI model considered for OFDM is a zero-mean Gaussian random process that occupies certain carriers along with white noise as

$$
S_{n}(k)=\left\{\begin{array}{ll}
\frac{N_{i}+N_{w}}{2}, & \text { if } k_{1}<k<k_{2} \\
\frac{N_{w}}{2}, & \text { otherwise }
\end{array},\right.
$$

where $k$ is the carrier index, and $\frac{N_{i}}{2}$ and $\frac{N_{w}}{2}$ are the power spectral densities of the narrowband interferer and white noise, respectively. In OFDM based UWB, NBI can be avoided easily by an adaptive OFDM system design. As the simple interference scenario illustrated in Fig. 2 shows, NBI will corrupt only some carriers in the OFDM spectrum. Therefore, only the information that is transmitted over these frequencies will be affected from the interference. If the interfered carriers can be identified, transmission over these carriers can be avoided. In addition, by sufficient FEC and frequency interleaving, jamming resistance against NBI can be obtained easily. Avoiding or adapting the transmission over the strongly interfered carriers can provide more spectrum and power efficiency, as these increase the immunity against NBI, and hence, relax the FEC coding power requirement.

In order to determine the interfered carriers, the noise temperature has to be estimated over the UWB-OFDM transmission band. In [10], a noise temperature estimation technique for UWB-OFDM systems is developed. In this technique, noise and interference are not interpreted as a single white noise term 


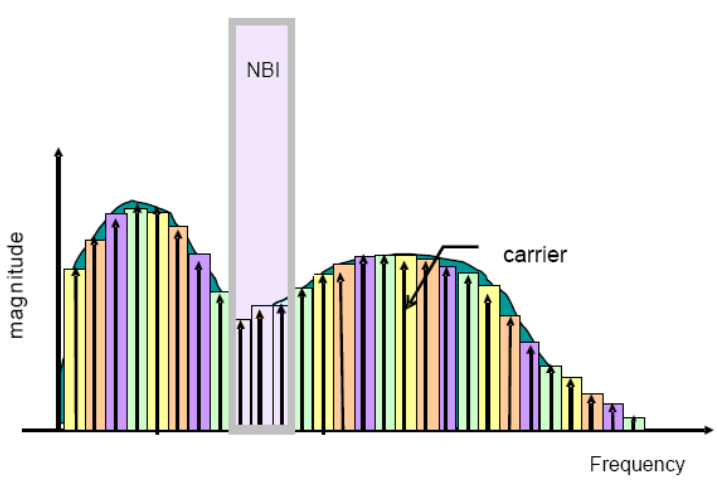

Fig. 2. A simple narrowband interference scenario for UWB-OFDM systems.

(which is the way they have been considered in the literature so far); instead, the color and the other statistics of the interference have been taken into account for improved interference temperature estimation. Conventional noise estimation algorithms assume that the noise statistics remain constant over the OFDM frequency band, and depending on this assumption, they average the instantaneous noise samples to get a single estimate. In reality, however, noise is often made up of white Gaussian noise along with correlated colored noise that affects the OFDM spectrum unevenly. In [10], for estimating the noise power an adaptive windowing technique is employed that takes into account the variation of the noise statistics across the OFDM carrier index as well as across OFDM symbols.

\section{COGNITIVE RADIO}

Traditional communication system design is based on allocating fixed amounts of resources to the user. Adaptive design methodologies, on the other hand, typically identify the requirements of the user, and then allocate just enough resources, thus enabling more efficient utilization of system resources and consequently increasing capacity. Pushing the adaptive system design further by introducing advanced attributes such as multidimensional awareness, sensing, as well as learning from its experiences to reason, plan, and decide on future actions to meet user needs leads to the cognitive radio concept. Ignited by the earlier work of Mitola [1], cognitive radio is a novel concept for future wireless communications, and it has been gaining significant interest among the academia, industry, and regulatory bodies [11].

Even though there is no consensus on the formal definition of cognitive radio, the concept has evolved recently to include various meanings in several contexts. One of its main aspects is related to autonomously exploiting locally unused spectrum to provide new paths to the spectrum access. Other aspects include

- inter-operability across several networks,

- roaming across borders, while being able to stay in compliance with local regulations,

- adapting the system, transmission, and reception parameters without user intervention,

- having the ability to understand and follow actions and choices of the users,

- and learning over time to become more responsive and to anticipate the user needs.

Cognitive radio concept proposes to furnish the radio systems with the abilities to measure and be aware of parameters related to the radio channel characteristics, availability of spectrum and power, interference and noise temperature, available networks, nodes, and infrastructures, as well as local policies and other operating restrictions. The primary advantage targeted with these features is to enable the cognitive systems to utilize the available spectrum in the most efficient way.

\section{OPPORTUNISTIC SPECTRUM USAGE}

Conventionally, frequency spectrum allocation for radio systems has been done in the form of licensing different frequency bands to separate applications. In this procedure, a licensed user possesses the absolute ownership of the spectrum it is allocated, and the spectrum can not be offered to the usage of other potential users, even if the licensed user is temporarily not making use of it. Therefore, the static frequency allocation leads to a highly poor utilization of the spectrum. It has been shown by the Spectrum Policy Task Force (SPTF) of the FCC that many licensed frequency bands are not being used for long durations [12]. Also, a recent experiment conducted in New York, United States in September 2004 revealed that the average duty cycle of the spectrum between $30 \mathrm{MHz}$ and $3 \mathrm{GHz}$ was only $13 \%$ [13].

Cognitive radio initiates a revolution regarding the spectrum allocation considerations by putting forward a new concept called opportunistic spectrum usage, which involves the soft usage of the current licensed and unlicensed available spectrum. This concept proposes that licensed bands can be utilized by secondary users at times when they are not being used by their owners, leading to the most efficient exploitation of the entire spectrum. In this opportunistic way of spectrum usage, it has to be guaranteed by the unlicensed systems that their operation does not affect the primary users. Although this approach is similar to the UWB from the point that both are unlicensed, there are two main differences between the opportunistic usage and UWB. First, UWB systems are forced to occupy a band of at least $500 \mathrm{MHz}$ width, which is not the case for the opportunistic usage; and second, for UWB communications there is a strict transmit power limitation, whereas in opportunistic usage the transmitted power can be comparable to the power of licensed systems.

\section{A. Sensing the Spectrum Opportunities}

A solid understanding of the opportunistic spectrum usage concept requires that opportunity is defined clearly. Cognitive radios periodically scan the spectrum and detect the spectra that are temporarily not being used by their licensed users, which can be called white bands. In many works, white bands are directly taken as the spectrum opportunities. However, there are spectral, temporal and spatial requirements that a white band has to satisfy in order to be useful and to be considered an opportunity [14], [15]. These requirements can be listed as follows. 
1. Opportunity is not an instantaneous white space in spectrum. It is necessary to monitor a white space continuously over a time frame (in the order of seconds) and ensure that it does not display an erratic behavior, i.e. for a reasonably long time the noise temperature in that band resides below a certain threshold, and the band remains as a white space.

2. It may not be reliable to consider a white band an opportunity if it is detected by only one single cognitive radio device. The reasons include that the device has a limited sensing range, as well as that it may be experiencing shadowing. Optimally, the spectrum has to be sensed by a number of cognitive nodes over a region that goes well beyond the range of a single cognitive device. A band can be considered a candidate for being a spectrum opportunity only if it is detected as white by many cognitive nodes at different locations that exchange the spectrum sensing information with each other.

3. If a frequency band to be utilized is too narrow, it may be hard for the cognitive radio to generate a temporally limited pulse shape that fits into that band. Therefore, a white band has to be wider than a certain bandwidth for being targeted for opportunistic spectrum usage.

Apparently, these rules are required to minimize the risk of causing interference to licensed systems. Beside this, such an opportunity definition is optimum from the point of minimizing the computational burden, as well, because it saves a cognitive radio from doing computations and changing its parameters without ensuring the dependability of a white band.

In the literature, there is a limited number of methods proposed regarding the implementation of spectral sensing for cognitive radio [2], [16], [17]. At the system level, spectral sensing can be implemented in an individual or distributed manner [18]. In the individual sensing, the cognitive UWB device senses the spectrum by its own means, and depends on this knowledge when making decisions. However, because of the definition of opportunity, it is not the preferred method for sensing. In the distributed sensing, which can be non-centralized or centralized, multiple devices scan the spectrum, and share the gathered information with each other. In non-centralized spectrum sensing, it is considered to have an allocated control channel to transmit this information [19]. In centralized sensing, on the other hand, it is contemplated to have a central controller that gathers this information, decides for spectrum availability, and allocates distinct bands to different cognitive users [18], [20].

\section{B. Spectrum Shaping}

A challenging requirement of opportunistic usage is that the cognitive transceiver has to be able to dynamically adapt its transmission parameters to operate over a wide range of spectrum with different bandwidths, which can be called spectrum shaping capability. Spectrum shaping is accomplished by modifying the transmitted power level and the pulse shape in such a way that the spectrum of the pulse fills the detected spectrum opportunities as efficiently as possible. Various methods to implement pulse shaping for cognitive radio are given in [21]-[25].

A possible option for filling the white spaces is to employ OFDM carriers as in the case of UWB-OFDM. OFDM based implementation of spectrum shaping can be found in [21] and [22]. However, when shaping the spectrum of the transmitted
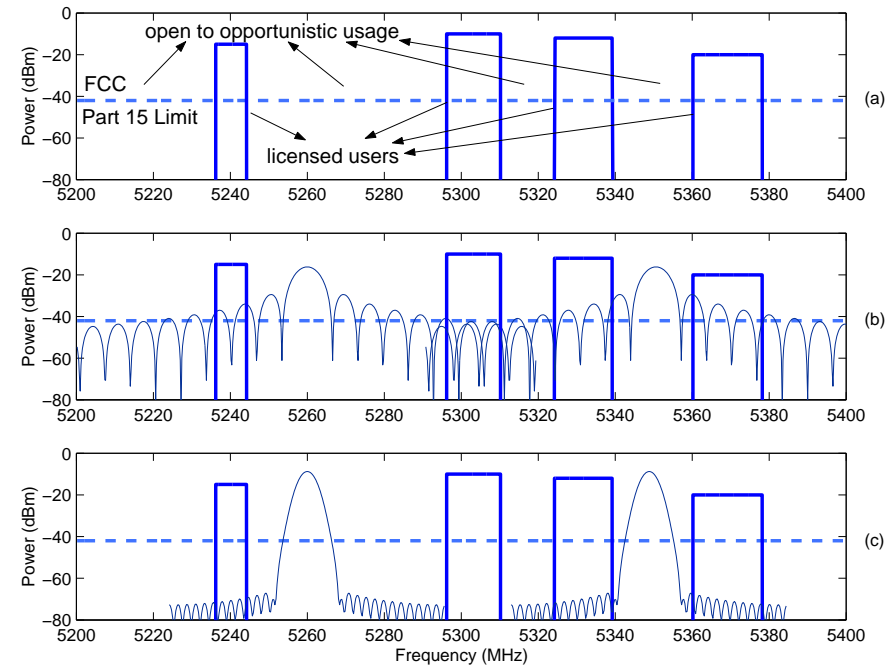

Fig. 3. a. A snap-shot of the spectrum in time. b. Opportunistic spectrum utilization employing time limited sinusoids. c. Opportunistic spectrum usage employing special pulses.

pulse, it has to be strictly ensured that the leakage from the opportunity bands to the licensed systems in the adjacent bands remains at a negligible level (illustrated in Fig. 3). If the transmitted pulses are time limited sinusoids (as in the case of OFDM) and no windowing is used, the resulting side lobes may be unacceptably high (see Fig. 4-a). Therefore, it is very important to employ special pulses that

- have sharp fall-offs and suppressed side lobes in the frequency domain

- are limited both in time and bandwidth,

- have a pulse width and bandwidth that can be controlled simultaneously.

Prolate Spheroidal Wavelet Functions (PSWF) satisfy these requirements to a large extent (shown in Fig. 4-d). Spectrum shaping methods employing the PSWF are provided in [23] and [24].

An alternative method for shaping the spectrum of the transmitted pulse is based on the usage of raised cosine (or root raised cosine) filters, which can be exemplified as in Fig. 4-b (and c). In this method, first, the center frequencies $f_{c_{i}}$ and bandwidths $B_{i}$ of each opportunity $O_{i}$ for $i=1,2, \ldots, N$, are determined, where $N$ is the total number of opportunities. In the next step, making use of its awareness property, the cognitive radio selects the raised cosine filters $r_{i}(t)$ that are the most suitable for each $O_{i}$. Filling a higher percentage of a white space requires a higher roll-off filter, which corresponds to a longer symbol in time, leading to inter-symbol interference or a lower throughput. Hence, the cognitive radio determines the filter to be used according to the amount of available bandwidth and the data rate required. The selected filters are multiplied with digitally generated cosine signals yielding

$$
\phi_{i}(t)=\cos \left(2 \pi f_{c_{i}} t\right) \cdot r_{i}(t) \text {. }
$$

$\phi_{i}(t)$ can be exemplified as in Fig. 5-a,b, and c, which are generated using $r_{i}(t)$ with roll-off coefficients $0.9,0.3$ and 0.5 , respectively. Each of these pulses is filling one of the opportunities in Fig. 5-e. The final pulse shape (demonstrated in Fig. 5-d) is 

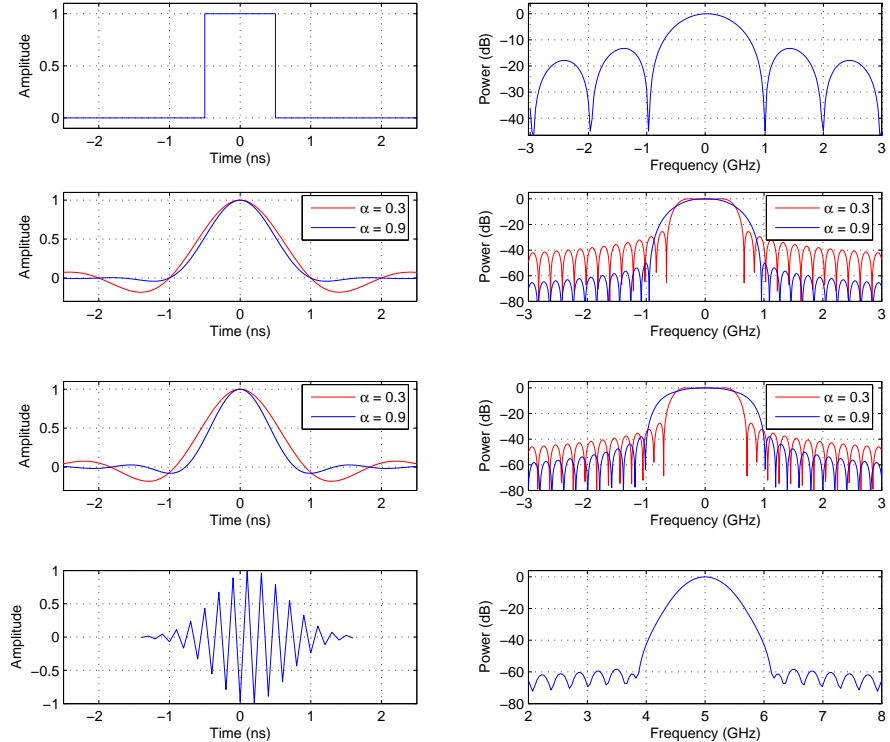

Fig. 4. Different pulse shapes and their spectra a. Rectangular window. b. Raised cosine windows with roll-off factors $\alpha=0.3$ and $\alpha=0.9$. c. Root raised cosine windows with roll-off factors $\alpha=0.3$ and $\alpha=$ 0.9 . d. A high order prolate spheroidal wavelet function.

obtained by taking the sum of all these separate pulses

$$
p(t)=\sum_{i=1}^{N} \phi_{i}(t)
$$

and it fills the opportunities as shown in Fig. 5-f.

The current transceivers include an analog front-end, which is mostly fixed for a specific function to operate over a small range of frequencies. Such an analog front-end is not flexible and not programmable. This gives rise to a new concept called software defined radio (SDR), where this fixed analog circuitry needs to be replaced with software programmable hardware [11]. The ideal SDR concept digitizes the received signal as soon as possible so that a flexible radio functionality can be obtained. As can be seen, this is a challenge with the current analog-to-digital-converter (ADC) capabilities and with the processing power available. Therefore, currently, the new generation wireless systems are slowly integrating a version of this concept.

\section{COGNITIVE UWB-OFDM}

Under the current FCC regulation, UWB systems are allowed to have a very limited transmit power. Hence, all current UWB efforts both from industry and academy are in the direction of making UWB systems work in an underlay scenario, and aim at wireless personal are networks (WPAN), only. In this paper, we consider cognitive UWB radios to be capable of switching between UWB and opportunistic spectrum usage, whichever is more advantageous.

In the UWB-OFDM communications that we contemplate, for UWB devices without cognitive capabilities, the power limitations specified with the published spectral masks will remain
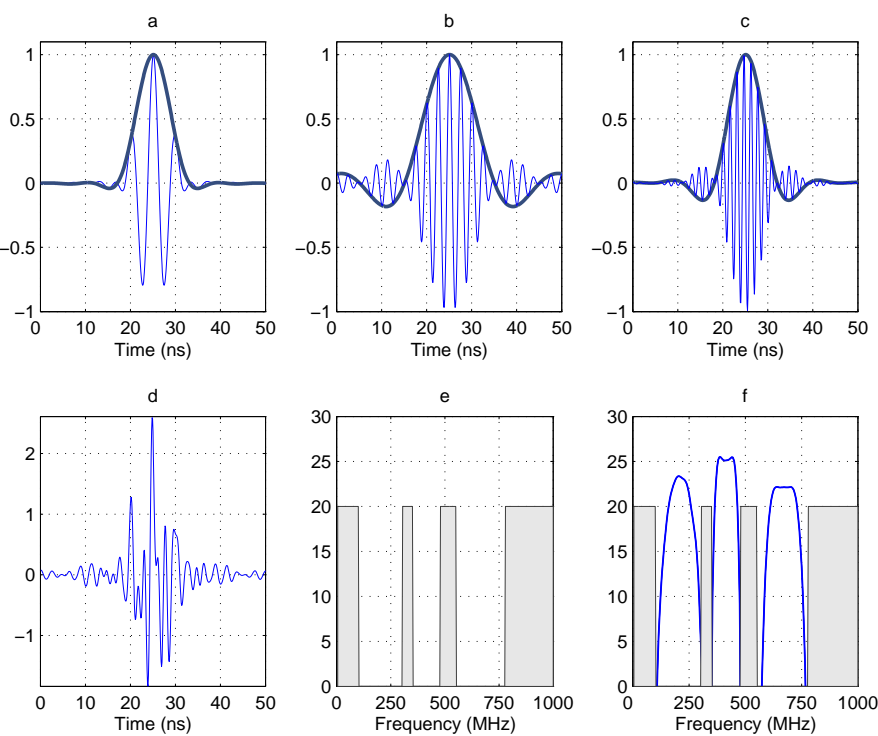

Fig. 5. a-,b-,c- Separate pulses obtained via raised cosine filtering that fit into different opportunities. $d$ - Sum of the separate pulses. e- Binary classification of frequency bands as 'occupied' or 'opportunity'. fSpectrum of the designed pulse filling the opportunities.

as they are. For the cognitive UWB radios, however, we expect the regulatory agencies to provide additional freedom for the transmitted power. A motivating example is the fact that the SPTF has already been considering alternative ways of allocating the spectrum [12]. By raising the power level, it is aimed to free the UWB devices from being restricted to short range applications.

Being able to implement both, cognitive UWB-OFDM systems decide between UWB-OFDM and opportunistic usage according to the conditions. One of the main decision criteria is that UWB-OFDM can make instant changes in the spectrum it occupies by turning on and off some carriers depending on the spectrum usage of licensed systems. Opportunistic usage, on the other hand, requires that a band is scanned by a number of cognitive radios, remains available for a certain time, and satisfies some spectral quality conditions, and therefore, it is not suitable for speedy changes.

Opportunistic usage may be especially attractive for applications that require a high quality-of-service (QoS) because of its high transmit power and wide band usage (relative to narrowband systems). Also, if the available bands, which may be targeted for either UWB-OFDM or opportunistic usage, are at high frequencies, the latter can be a better option because of the higher path loss at these frequencies.

Although, in general, opportunistic usage seems to be more advantageous and desirable than UWB-OFDM, in certain scenarios, such as the ones listed below, cognitive UWB radios may have to select UWB-OFDM.

- If some primary users have a frequency hopping signal, and hence, the spectral conditions are changing very fast, the cognitive radio may not able to keep track of the spectrum opportunities and it can switch to UWB-OFDM.

- If the primary user is time hopping, cognitive radio might need to monitor the spectrum for an extra long time frame and 
may still not determine the timing sequence, or may be unable to adapt itself to continuously changing spectrum.

- If the primary user is mobile (or steadily moving in a certain area), it may be risky to use the spectrum opportunistically because the communication of the primary system can be easily disturbed. Hence, UWB-OFDM can be employed.

- The bands open to opportunistic usage may be too much divided by narrowband systems (into a number of separated narrow bands), leading to extra long pulses in time. Again, in this case UWB-OFDM can be preferable.

- If numerous licensed users join and leave the spectrum in a frequent manner (like in a GSM band), opportunistic usage may not be feasible.

- Opportunistic usage requires setting a threshold in order to determine whether a certain band is occupied. If the noise floor in a band is changing continuously, it may not be possible to determine a reliable threshold, and UWB-OFDM may be preferred.

- Gray bands (the bands in which the noise temperature is not as low as in the white bands) can be a potential target for UWBOFDM.

- If the spectrum sensing results from different nodes in a cognitive network do not match to a large extent, this may indicate that either some of the nodes are being shadowed (and can not detect primary users), or the spectrum sensing information of some nodes can not be transmitted (or detected) correctly. In both cases, as a precaution, switching to UWB-OFDM can be reasonable.

- Even if there is an adequate amount of spectrum open to opportunistic usage, the number of cognitive users that target at this spectrum might be too high, forcing some of these users to switch to UWB-OFDM.

\section{CONCLUSION}

The continuously increasing need for frequency spectrum requires to increase the efficiency of spectrum usage. Therefore, it is necessary to develop flexible and adaptable radio access technologies that can take advantage of the available spectrum in an opportunistic way. In this paper, UWB-OFDM is shown to be an attractive technology because of its various adaptation capabilities and its resistance against interference caused by licensed systems. The opportunity concept is defined in detail, and regarding the opportunistic spectrum usage, opportunity sensing and spectrum shaping topics are investigated. It is shown that the marriage of OFDM based UWB with opportunistic spectrum usage will open the doors for further improvements in spectral efficiency, and bring about concepts that will allow the joint underlay and overlay usage of the spectrum. Although this comes at the expense of increased hardware complexity relative to pure UWB, it is made clear that the advantages of cognitive UWBOFDM would pay off for this increase.

Sensing the licensed users and searching for opportunity across multiple dimensions is a wide research area for academic and industrial communities. UWB, which is transmitting over a wide bandwidth, along with the UWB receivers, which are already designed with the capability of capturing anything within the transmission bandwidth, can also be equipped to process, measure, and sense the primary users over the transmission bandwidth without the need of additional units. Not only the noise temperature, but also additional statistical and deterministic information about the primary users can be extracted with the OFDM based UWB technology. Shaping the power spectrum of the transmitted signal while taking advantage of the spectral opportunities as efficiently as possible without creating any disturbance to the primary users is another tempting research area.

UWB, OFDM, and cognitive radio are terms that the wireless community has already been heavily exposed to over the recent years. It is our anticipation that in the near future, the wireless community will be encountering that these terms are mentioned jointly in the context of the spectrum efficiency and opportunistic spectrum usage.

\section{REFERENCES}

[1] J. Mitola, "Cognitive radio for flexible mobile multimedia communications," in Proc. Mobile Multimedia Commun. (MoMuC '99), pp. 3-10, Nov. 1999.

[2] S. Haykin, "Cognitive radio: Brain-empowered wireless communications," IEEE J. Select. Areas Commun., vol. 23, pp. 201-220, Feb. 2005

[3] S. Mangold, Z. Zhong, K. Challapali, and C.-T. Chou, "Spectrum agile radio: radio resource measurements for opportunistic spectrum usage," in Proc. IEEE Global Telecomm. Conf. (GLOBECOM), vol. 6, (Dallas, TX), pp. 3467 - 3471, Dec. 2004.

[4] J. Foerster, "Ultra-wideband technology enabling low-power, high-rate connectivity (invited paper)," in Proc. IEEE Workshop Wireless Commun. Networking, (Pasadena, CA), Sep. 2002.

[5] J. R. Foerster, "The performance of a direct-sequence spread ultrawideband system in the presence of multipath, narrowband interference, and multiuser interference," in Proc. IEEE Vehic. Technol. Conf., vol. 4 (Birmingham, AL), pp. 1931-1935, May 2002.

[6] J. Choi and W. Stark, "Performance of autocorrelation receivers for ultrawideband communications with PPM in multipath channels," in Proc. IEEE Ultrawideband Syst. and Technol. (UWBST), (Baltimore, MD), pp. 213 -217, May 2002.

[7] L. Zhao and A. Haimovich, "Performance of ultra-wideband communications in the presence of interference," IEEE J. Select. Areas Commun., vol. 20 , pp. 1684-1691, Dec. 2002.

[8] G. Durisi and S. Benedetto, "Performance evaluation of TH-PPM UWB systems in the presence of multiuser interference," IEEE Commun. Lett., vol. 7, pp. 224-226, May 2003.

[9] R. Tesi, M. Hamalainen, J. Iinatti, and V. Hovinen, "On the influence of pulsed jamming and coloured noise in UWB transmission," in Proc. Finnish Wireless Commun. Workshop (FWCW), (Espoo, Finland), May 2002.

[10] T. Yücek and H. Arslan, "MMSE noise power and SNR estimation for OFDM systems," in Proc. IEEE Sarnoff Conf., (Princeton, NJ), March 2006. Accepted for publication.

[11] W. D. Horne, "Adaptive spectrum access: Using the full spectrum space," in Proc. 31st Annual Telecommunications Policy Research Conference (TPRC 03), Oct. 2003

[12] Federal Communications Commission, "Spectrum Policy Task Force Report ET Docket no. 02- 135,” Nov. 2002.

[13] "Spectrum Occupancy Report for New York City during the Republican Convention August 30 - September 1, 2004,” Jan. 2005.

[14] M. P. Olivieri, G. Barnett, A. Lackpour, A. Davis, and P. Ngo, "A scalable dynamic spectrum allocation system with interference mitigation for teams of spectrally agile software defined radios," in Proc. IEEE Int. Symp. Dynamic Spectrum Access Networks (DySPAN) 2005, (Baltimore, MD), pp. 170-179, Nov. 2005.

[15] H. Zheng and L. Cao, "Device-centric spectrum management," in Proc. IEEE Int. Symp. Dynamic Spectrum Access Networks (DySPAN) 2005. (Baltimore, MD), pp. 56-65, Nov. 2005.

[16] T. A. Weiss and J. H. F. K. Jondral, "A diversity approach for the detection of idle spectral resources in spectrum pooling systems," in Proc. 48th Int. Sci. Colloq., (Illmenau, Germany), Sep. 2003.

[17] D. Cabric, S. M. Mishra, and R. W. Brodersen, "Implementation issues in spectrum sensing for cognitive radios," in Proc. IEEE Asilomar Conf. on Signals, Syst., Computers, vol. 1, (Pacific Grove, CA), pp. 772-776, Nov. 2004.

[18] J. Hillenbrand, T. Weiss, and F. Jondral, "Calculation of detection and false alarm probabilities in spectrum pooling systems," IEEE Comm. Letters, vol. 9, pp. 349-351, April 2005. 
[19] X. Jing and D. Raychaudhuri, "Spectrum co-existence of IEEE $802.11 \mathrm{~b}$ and 802.16a networks using the CSCC etiquette protocol," in Proc. IEEE Int. Symp. Dynamic Spectrum Access Networks (DySPAN) 2005, (Baltimore, MD), pp. $243-250$, Nov. 2005.

[20] G. Ganesan and Y. Li, "Cooperative spectrum sensing in cognitive radio networks," in Proc. IEEE Int. Symp. Dynamic Spectrum Access Networks (DySPAN) 2005, (Baltimore, MD), pp. 137 - 143, Nov. 2005.

[21] T. A. Weiss and F. K. Jondral, "Spectrum pooling: an innovative strategy for the enhancement of spectrum efficiency," IEEE Commun. Mag., vol. 42, pp. 8-14, Mar. 2004.

[22] J. Poston and W. Horne, "Discontiguous OFDM considerations for dynamic spectrum access in idle TV channels," in Proc. IEEE Int. Symp. Dynamic Spectrum Access Networks (DySPAN) 2005, (Baltimore, MD), pp. $607-610$, Nov. 2005.

[23] R. Dilmaghani, M. Ghavami, B. Allen, and H. Aghvami, "Novel UWB pulse shaping using prolate spheroidal wave functions," in Proc. IEEE Int. Symp. on Personal, Indoor and Mobile Radio Commun. PIMRC 2003, vol. 1, (Beijing, China), pp. 602 - 606, Sept. 2003.

[24] K. Wallace, B. Parr, B. Cho, and Z. Ding, "Performance analysis of a spectrally compliant ultra-wideband pulse design," IEEE Trans. on Wireless Commun., vol. 4, pp. 2172-2181, Sept. 2005.

[25] M. G. Di Benedetto and L. De Nardis, "Tuning UWB signals by pulse shaping," Special Issue on Signal Proc. in UWB Commun., Eurasip Journal on Signal Proc., Elsevier Publishers, 2005. to appear.

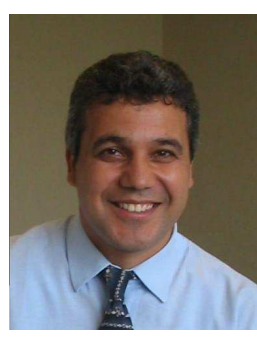

Dr. Hüseyin Arslan has received his Ph.D. degree in 1998 from Southern Methodist University (SMU), Dallas, TX. From January 1998 to August 2002, he was with the research group of Ericsson Inc., NC, USA, where he was involved with several project related to $2 \mathrm{G}$ and $3 \mathrm{G}$ wireless cellular communication systems. Since August 2002, he has been with the Electrical Engineering Dept. of University of South Florida. He has also been working for Anritsu Company, Morgan Hill, CA (as a visiting professor during the summer of 2005) as a part-time consulting since August 2005. His research interests are related to advanced signal processing techniques at the physical layer, with cross-layer design for networking adaptivity and Quality of Service (QoS) control. He is interested in many forms of wireless technologies including cellular, wireless PAN/LAN/MANs, fixed wireless access, and specialized wireless data networks like wireless sensors networks and wireless telemetry. The current research interests are on UWB, OFDM based wireless technologies with emphasis on WIMAX, and cognitive and software defined radio. He has served as technical program committee member, session and symposium organizer in several IEEE conferences. He is editorial board member for Wireless Communication and Mobile Computing journal, and was technical program co-chair of IEEE wireless and microwave conference 2004. Dr. Arslan is a senior member of IEEE.

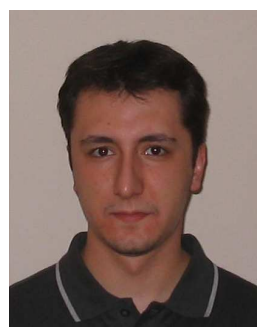

Mustafa Emin Şahin was born in Istanbul, Turkey, on September 7, 1981. He received his B.S. degree in Electrical and Electronic Engineering from Bog̃aziçi University, Istanbul, Turkey, in June 2004, and his M.S. degree in Electrical Engineering from the University of South Florida, Tampa, FL, USA, in April 2006. He is a member of the Wireless Communications and Signal Processing Group at University of South Florida and he is working toward his Ph.D. degree. His research interests are spectrum sensing and spectrum shaping for cognitive radio, energy detector receivers for impulse radio based ultrawideband systems, and narrowband interference and inter-symbol interference effects in UWB communications. He is a student member of IEEE. 